\title{
Subject Index, Vol. 29, 1997
}

Absorption 246

Acute allograft rejection 12

- pancreatitis 382

Adenosine triphosphate 438

Adhesion molecules 160

$\alpha$-Adrenergic blockade 20

Allopurinol 101,429

Anastomosis, conventional end-

end 75

Animal model 124

Anismus 42

Antagonist 133

Anti-rat intercellular adhesion molecule-1 monoclonal antibody 93

Antioxidants 368

Aortic clamping 133

Apoptosis 396

Ascorbic acid 187

Atrial natriuretic peptide 124

Bacterial translocation 187

bcl-2 396

Bile duct reconstruction 421

Biopsy 292

Blood cardioplegic solution 20

- flow 438

Bone histomorphometry 209 Branched chain amino acids 382 Bretschneider's HTK solution 264 Bromodeoxyuridine test 4215 'Bromo-deoxy-uridine 411

Carcinoembryonic antigen 465 Catecholamines 124 CD18 160 CD62L 160 Cell culture 292 Cholecystectomy 27 Cholecystitis 150 Cholecystokinin-octapeptide 362 Choledochocholedochostomy 195 Cholestatic j aundice 187 Cisplatin 396 Colon 447

- cancer 473

Colorectal tumors 465 Complications, morphological 75 Computer-assisted surgery 481 Constipation 42 Contraction force 254

Coronary flow 229

- vascular resistance 20

Cortisol 27

Cystic duct occlusion 150 Cytokines 27

D2 resection 303 Descending relaxation 311 Dibutyryl cyclic adenosine mono-phosphate 438 Diltiazem 229 Distribution 411

DNA index 465

- patterns 465

Donor nutritional status 339 Duodenal growth 142 Duplex sonography 455

Early gastric cancer 35

Endothelial cell monolayer 375

Endothelin 124

Endotoxemia 237

Epidermal growth factor 142, 222,

447 Experimental study, mice 150 Extracorporeal heart-lung model

5,327 
Fascial healing 84 Fat embolism 124 Fecal peritonitis $390 \alpha$-Fetoprotein 465 Fibroblasts, cultured 84 Flush perfusion 5 Frameless stereotaxy 481

Gadolinium chloride 176,375 Galactose elimination capacity

346 Gastrectomy 209 -, total 303

Gastric cancer 287

- - cells 396

- mucosal glycoproteins 280 Gastrointestinal motility 311 Glucagon 339 Glutathione 264 Glycogen content 339

Graft vascularization 457 Gross ulcer index 280 Gut 246

Heart transplantation 229 Hemodynamics 124 Hemoperfusion 356 Hepatectomy, partial 273 Hepatic allograft 107

- $\quad$-, rat 116

- ischemia-reperfusion injury 93

- tissue blood flow 93 Hepatocyte transplantation, intra-

portal 411 Heterogeneity 403 HIDA clearance 346 High-energy phosphates 101 Histomorphometric evaluation

280 Hydatidcyst 202

- relapse 202

Hypoxemia, pulmonary 124

Idiotype 116 Ileocecal valve 75 Image-guided neurosurgery 481 Implantation 473 Infiltrating cells 107 Initial cell loss 411 Integrins 160 Interleukins 27 Intestine 246 Isch(a)emia 101,246 -, reperfusion 346 Ischemia-reperfusion injury 254

- - , hepatic 429

Isolated rabbit distal colon 311

KA R.G Ell C 1997 S. Karger AG,

491

E-Mail karger@karger.ch Fax +41613061234 http://www. karger. ch

Ki-67 35 Killer T cells 107 Knot-breaking strength 52 Knot-holding capacity 52 Kupffer cell function 356

Laparoscopy 27

Linear tensile strength 52

Lipid peroxidation 429

- peroxides 438 Liver 273

- circulation 237

- function 346

- macrophage 176

- metabolism 237

- neutrophil sequestration 93

- regeneration 273 Low potassium 327

Lung preservation $1,5,319,327$

- transplantation 1,12,319,327

Lymph node metastasis 35

Lymphadenectomy 303

Lymphocyte phenotyping 465

Lymphokine 116

MAGE-1 403

Melanoma 403

Methylene blue 390

Mineral metabolism 209

Motility index 311

MR spectroscopy 101

Mucosal hexosamine content 280

- thickness 447

Muscle flap 42

Natural killer cells 107 Neuronavigation 481 Neuropeptide 362 Neurotensin 362 New Zealand white rabbit 311 Nitric oxide 237 Nonadrenergic intrinsic neural

component 362 Noncholinergic intrinsic neural 
component 362

Obturator internus 42 Octreotide 311

Orthotopic rat liver transplantation 421 Oxidative stress 429 Oxygen radical 429 Oxygen-free radical(s) 368,438

p53 35,396 Pancreas-preserving total gastrec-

tomy 287 Pancreatic blood flow 287

- growth 142 Perfusate temperature 5 Perfusion, transplant 12 Permeability 375 Platelet-activating factor 133 Pneumocytes

type II 319 Polyester vascular grafts 368 Polyglycolic acid mesh 84 Portal hypertension 187,280

- veinligation 273 Postgastrectomy bone disease 209 Postischemic shock 133 Potassium-reduced solutions 327 Povidone-

iodine 473 Preservation 246

- solutions 327 Processing 292

Quantification 411

Rat(s) 195,209,264 -, syngeneic 411 Rejection 229 Reperfusion 101, 133,246 Retrograde preservation 1 Reversible obstructive jaundice

195 Rewarming 339 Robotic surgery 481

Scolicidal agents 202 Selectin 160 Sepsis 160, 237

Septic shock syndrome 390 Skeletal muscle 101, 254, 438 Small bowel 246

- $\quad$ - transplantation 362

- intestine 447 Sodium nitroprusside 237 Splenectomy 176, 303 Splint technique 421 Stomach 447

Storage solution 319 Subendocardial hypoperfusion 20 Substance P 362 Superoxide dismutase 368 Suppressive factor 116 Suture material 473

Thyroid hormones 222 Thyroidectomy $222 \alpha$-Tocopherol 187 Total parenteral nutrition 382 Transbronchial biopsy 12

Transplantation 455 Tritiated thymidine 447 Tumor 292

- $\quad$ necrosis factor 176,375

- - factor- $\alpha 27$

University of Wisconsin solution 264

Vaccination 292

Valve substitutes, experimental

75 Vascular tone 124 Vasoconstriction 124

Working heart 264

${ }^{\mathrm{B} 3}$ Xe radiospirometry 12 Xenotransplantation 356

492

Eur Surg Res Vol. 29, 1997

Subject Index 